\title{
Recent advances in thoracic x-ray computed tomography for pulmonary imaging
}

\author{
Bruce J Precious MD, Rekha Raju MD, Jonathon Leipsic MD
}

BJ Precious, R Raju, J Leipsic. Recent advances in thoracic $\mathrm{x}$-ray computed tomography for pulmonary imaging. Can Respir J 2014;21(5):307-309.

The present article reviews recent advances in pulmonary computed tomography (CT) imaging, focusing on the application of dual-energy CT and the use of iterative reconstruction. Dual-energy CT has proven to be useful in the characterization of pulmonary blood pool in the setting of pulmonary embolism, characterization of diffuse lung parenchymal diseases, evaluation of thoracic malignancies and in imaging of lung ventilation using inhaled xenon. The benefits of iterative reconstruction have been largely derived from reduction of image noise compared with filtered backprojection reconstructions which, in turn, enables the use of lower radiation dose CT acquisition protocols without sacrificing image quality. Potential clinical applications of iterative reconstruction include imaging for pulmonary nodules and high-resolution pulmonary CT.

Key Words: Dual-energy CT; Iterative reconstruction; Pulmonary imaging

$\Delta$ mong the recent advances in pulmonary imaging with commong the recent advances in pulmonary imaging with com-
puted tomography (CT), the most exciting developments have occurred in the application of dual-energy CT and the use of iterative reconstruction.

While the concept of dual-energy CT was first described and reported in the 1970s, its clinical use has only recently become possible due to improvements in CT temporal resolution and computational abilities. Single-source scanners with fast kilovoltage switching, dual-layered detectors and dual-source multidetector CT are all capable of acquiring images at different tube energy levels $(\mathrm{kEv})$, which permits discrimination of materials based on their unique interactions with photons of different $\mathrm{x}$-ray energies (1). Currently, clinical applications of dual-energy CT in thoracic imaging using iodine-based contrast media include characterization of pulmonary blood pool in the setting of pulmonary embolism (PE), characterization of diffuse lung parenchymal diseases and evaluation of thoracic malignancies (2). Imaging of lung ventilation using dual-energy CT and inhaled xenon gas is also being explored.

Dual-energy CT can be accomplished using various CT systems. Dual-source CT scanners (Siemens Medical Solutions, USA) are equipped with two $\mathrm{x}$-ray tubes and two detectors that operate independently, allowing registration of high $(140 \mathrm{kVp})$ and low $(80 \mathrm{kVp}) \mathrm{x}$-ray spectra. Single-source CT scanners use rapid kilovoltage switching technology (GE Healthcare, USA) to acquire dual-energy data by rapidly alternating the tube energy from low $(80 \mathrm{kVp})$ to high $(140 \mathrm{kVp})$. In this system, post-processing enables the selective creation of images as if they had been acquired from a single high or low monochromatic energy source $(\mathrm{kEv})$ unlike the traditional polychromatic source used for CT imaging (2). A third system not yet in routine clinical use employs layered detectors (Philips Healthcare, USA) that absorb lowor high-energy photons depending on their proximity to the single source, thus resulting in separate data sets used to create corresponding image series (1).

\section{Les récents progrès de la tomodensitométrie pulmonaire}

Le présent article passe en revue les récents progrès de la tomodensitométrie (TMD) pulmonaire et traite de TMD en double énergie et de reconstruction itérative. L'utilité de la TMD en double énergie est démontrée pour caractériser la ventriculographie en cas d'embolie pulmonaire, caractériser les maladies parenchymateuses diffuses des poumons, évaluer les cancers thoraciques et obtenir une imagerie de la ventilation pulmonaire à l'aide de xénon inhalé. Les avantages de la reconstruction itérative sont dérivés en grande partie de la réduction du bruit image par rapport aux reconstructions par rétroprojection filtrée. Ce type de reconstruction permet d'utiliser les protocoles de TMD à faibles doses de radiation sans sacrifier la qualité de l'image. Ses applications cliniques potentielles incluent l'imagerie des nodules pulmonaires et la TMD pulmonaire à haute résolution.

Clinical application of dual-energy CT technology centres on the basic principle of material decomposition enabled by attenuation differences at differing energy levels. The mean photon energy at $80 \mathrm{kVp}$ is slightly above the $\mathrm{K}$-shell binding energy of iodine, which maximizes photoelectric absorption of iodine and permits its differentiation from other materials (2). In the lungs, dual-energy post-processing software can perform material decomposition selective for iodine to create perfusion maps of the lung parenchyma or to remove iodine for virtual noncontrast images. Material decomposition selective for thrombus can be used for characterization of soft tissue within the pulmonary arteries (1). Similarly, inhaled xenon can be differentiated to perform ventilation mapping.

Application of dual-energy CT in the evaluation of acute PE has gained considerable interest. While conventional pulmonary CT angiography (CTA) provides morphological information used for the diagnosis of PE, dual-energy CTA provides simultaneous assessment of the effects of thrombus on pulmonary perfusion, which may alter patient management, risk stratification and prognostication (1). Fink et al (3) reported that the sensitivity and specificity of dual-energy CT perfusion, compared with conventional CTA in the evaluation of $\mathrm{PE}$, were both $100 \%$ on a per-patient basis and $60 \%$ to $66.7 \%$ and $99.5 \%$ to $99.8 \%$, respectively, on a per-segment basis. Dual-energy pulmonary perfusion imaging has also been reported to increase the sensitivity for detection of small thrombi in segmental or subsegmental pulmonary arteries (4). Dual-energy-based blood imaging may also be useful in the follow-up of PE to assess for improvement in perfusion defects following anticoagulation therapy (2). Dual-energy CTA of the lungs can be used to evaluate vascular characteristics of chronic PE, such as to demonstrate the typical mosaic pattern of blood pool distribution in chronic $\mathrm{PE}$, as opposed to the wedge-shaped appearance of acute PE, to assess for collateral vascular supply distal to pulmonary arterial obstruction and to demonstrate calcification in chronic clots in the setting of pulmonary hypertension using virtual noncontrast imaging (5).

St Paul's Hospital, Vancouver, British Columbia

Correspondence: Dr Bruce J Precious, 2217 Balsam Street, Vancouver, British Columbia V6K 4N2.

Telephone 902-225-1588, fax 604-806-8437, e-mail bpreciou@dal.ca 
Evaluation of the regional severity of emphysema can also be performed using dual-energy CT, which can simultaneously provide highresolution anatomical imaging and functional perfusion imaging in a single acquisition. Lee et al (6) showed moderate correlation between pulmonary function testing and the severity of pulmonary parenchymal destruction on virtual noncontrast imaging and diminished perfusion status on the iodine mapping acquired simultaneously.

The ability of dual-energy CT to simultaneously provide virtual non-contrast- and iodine-enhanced series from a single scan acquisition can also be applied to the evaluation of solitary pulmonary nodules by allowing measurement of the degree of enhancement and detecting calcium. Chae et al (7) showed that the diagnostic accuracy for distinguishing malignancy from benignity using CT values with a cut-off of 20 Hounsfield units on dual-energy-based iodine-enhanced images was comparable with that of using the degree of enhancement on pre- and postcontrast images (sensitivity $92 \%$ and $72 \%$; specificity $70 \%$ and $70 \%$; accuracy $82.2 \%$ and $71.1 \%$, respectively) (7). The virtual noncontrast images had the same diagnostic quality as the true noncontrast-weighted average images in $77.6 \%$ of their patients, whereas $8.2 \%$ were considered to be unacceptable and $16.3 \%$ were considered to be inferior yet diagnostic. Thus, the authors concluded that a 3 min postcontrast dual-energy study alone may be sufficient to characterize pulmonary nodules.

Other oncological applications of dual-energy CT equally show promise. Schmid-Bindert et al (8) showed moderate correlation between maximum standardized uptake value (SUVmax) of ${ }^{18} \mathrm{FDG}$ positron emission tomography (PET)-CT and iodine-related attenuation of dual-energy CT of primary tumours and ${ }^{18}$ FDG PET-CTpositive thoracic lymph nodes in patients with lung cancer.

Dual-energy CT using nonradioactive xenon for the assessment of ventilation has been gaining attention. Xenon is an inert radiodense gas, which has similar characteristics to iodine in terms of photoelectric absorption, allowing it to be used as a contrast agent for CT. The advantage of dual-energy CT imaging is that it enables separation of xenon from lung tissue using a single imaging acquisition followed by material decomposition, rather than having to image at multiple time points during dynamic washing in and washing out of the gas. With xenon-enhanced dual-energy CT, ventilation maps of the lung can be generated to assess ventilation function in patients with emphysema, asthma, and bronchial atresia or obstruction (5).

With growing concerns about increased radiation exposure from CT imaging, there has been significant focus aimed at reducing radiation exposure while maintaining diagnostic image quality. Until recently, clinical CT used filtered backprojection reconstruction techniques, mainly due to its limited computational requirements and speed. Filtered backprojection is an analytical reconstruction algorithm that uses the solution of an integral equation by inversion to propagate measured projection data into images in combination with a filter component. Traditional filtered backprojection reconstruction algorithms suffered from the trade-off between radiation dose and image noise (9). With progressive improvement of computing power, the use of iterative reconstruction algorithms has become possible with clinically acceptable reconstruction times, opening another avenue for dose savings. Iterative reconstruction techniques rebuild images by focusing on noise reduction. The exact techniques vary among vendors and generations. As a representative example, one type of iterative reconstruction technique uses matrix algebra to transform the measured value of each pixel to a new estimate of the pixel value, which is then correlated with an ideal value that a noise model predicts. This process is repeated in a succession of iterative steps until there is convergence of the final estimated and the ideal pixel values. In essence, noise is selectively removed from the image. Compared with filtered backprojection, iterative reconstruction enables acquisition of data using lower radiation exposure with a relatively lesser increase in image noise.

Similar to dual energy, there are several available iterative reconstruction algorithms, which vary slightly from vendor to vendor. Dose reductions, when compared with the conventional filtered backprojection reconstructions on routine chest CT, have been consistently reported, ranging from $27 \%$ to $80 \%$, without compromise of image quality (10). Neroladaki et al (11) showed that detection of pulmonary nodules was possible with radiation dose reductions to levels similar to conventional chest radiography using a second-generation model-based iterative reconstruction algorithm (Veo, GE Healthcare, USA) (11). Similarly, Xu et al (12) showed that model-based iterative reconstruction effectively reduces image noise compared with conventional filtered backprojection reconstructions, such that detection and quantification of ground-glass opacities on a phantom model was acceptable using ultralow-dose CT scanning. Quantification of pulmonary nodules in vivo was studied by Willemink et al (13), who showed that measurements of solid pulmonary nodule volumes with iterative reconstruction were comparable with conventional filtered backprojection regardless of the tube voltage and tube current-time product exposure settings, with no significant differences between measured volumes of both small and large solid nodules. Yanagawa et al (14) showed that the sensitivity of computer-assisted detection of pulmonary nodules using iterative reconstruction on lower-dose CT is almost equal to that using filtered backprojection on clinical routinedose CT (14).

In a prospective randomized study using an adaptive statistical iterative reconstruction (ASIR), Singh et al (15) showed that subjective and objective image noise could be significantly reduced to acceptable levels while achieving a lower radiation dose using $40 \mathrm{mAs}$ for chest CT. This was in contrast to an unacceptable increase in both subjective and objective noise on filtered backprojection images when radiation dose was decreased by lowering tube current from $110 \mathrm{mAs}$ to $75 \mathrm{mAs}$. The authors suggested a dose reduction of up to $75 \%$ for chest CT images reconstructed with ASIR with preserved image quality.

In high-resolution pulmonary CT imaging, iterative reconstruction has also been shown to result in equivalent or superior image quality compared with conventional filtered backprojection (10). Prakash et al (16) showed that the use of iterative reconstruction resulted in superior visualization of small anatomical details, such as interlobular septa, the centrilobular region and small airways, as well as abnormal findings, such as reticulations, tiny nodules, altered attenuation and bronchiectasis, compared with conventional filtered backprojection (16).

Recently, Nishio et al (17) showed that ASIR using threedimensional processing in low-dose CT scans resulted in greater consistency of emphysema quantification than the filtered backprojection, which is attributed to image noise effects on emphysema measurement reproducibility (17). However, there has been concern that with reduction of the radiation dose and resultant decrease in the signal-tonoise ratio, quantitative measurements may become unreliable. While several studies have shown that radiation dose can be lowered without clinical impact, others have demonstrated a significant difference in quantitative emphysema measurements as a result of dose reduction (18). Overall, it would appear that low-dose CT imaging is feasible for quantitative assessment provided scanning protocols are accounted for when comparing results between studies and over time (18).

Significant advancements have been realized recently with the integration of iterative reconstruction techniques and its resultant dose reduction and, in addition, the advent of dual-energy CT facilitating an element of functional assessment in pulmonary CT. While dual-energy CT is currently is used to a greater extent in the research arena, it offers exciting possibilities for clinical use in the realm of characterization of pulmonary blood pool in the setting of PE, characterization of diffuse lung parenchymal diseases, evaluation of thoracic malignancies and in imaging of lung ventilation using inhaled xenon. The benefits of iterative reconstruction have been largely derived from reduction of image noise compared with filtered backprojection reconstructions, which in turn enables the use of lower radiation dose CT acquisition protocols without sacrificing image quality. With progressive 
improvement in computational power, the use of iterative reconstruction algorithms has become possible with more reasonable reconstruction times. It is expected that, over time, iterative reconstruction will largely supplant filtered backprojection as the preferred method of image reconstruction. This opens promising avenues for dose savings across a broad range of clinical applications.

\section{REFERENCES}

1. Ko JP, Brandman S, Stember J, Naidich DP. Dual-energy Computed Tomography Concepts, Performance, and Thoracic Applications. J Thorac Imaging 2012;27:7-22.

2. Kang MJ, Park CM, Lee CH, Goo JM, Lee HJ. Dual-energy CT: Clinical applications in various pulmonary diseases. Radiographics 2010;30:685-98.

3. Fink C, Johnson TR, Michaely HJ, et al. Dual-energy CT angiography of the lung in patients with suspected pulmonary embolism: Initial results. Rofo 2008;180:879-83.

4. Lu GM, Wu SY, Yeh BM, Zhang LJ. Dual-energy computed tomography in pulmonary embolism. Br J Radiol 2010;83:707-18.

5. Lu GM, Zhao Y, Zhang LJ, Schoepf UJ. Dual-energy CT of the lung. AJR Am J Roentgenol 2012;199:S40-S53.

6. Lee CW, Seo JB, Lee Y, et al. A pilot trial on pulmonary emphysema quantification and perfusion mapping in a single-step using contrast-enhanced dual-energy computed tomography. Invest Radiol 2012;47:92-7.

7. Chae EJ, Song JW, Seo JB, Krauss B, Jang YM, Song KS. Clinical utility of dual-energy CT in the evaluation of solitary pulmonary nodules: Initial experience. Radiology 2008;249:671-81.

8. Schmid-Bindert G, Henzler T, Chu TQ, et al. Functional imaging of lung cancer using dual energy CT: How does iodine related attenuation correlate with standardized uptake value of ${ }^{18}$ FDGPET-CT? Eur Radiol 2012;22:93-103.

9. Wang H, Tan B, Zhao B, Liang C, Xu Z. Raw-data-based iterative reconstruction versus filtered back projection: Image quality of low-dose chest computed tomography examinations in 87 patients, Clin Imaging 2013;37:1024-32.

10. Leipsic J, Labounty TM, Heilbron B, et al. Estimated radiation dose reduction using adaptive statistical iterative reconstruction in coronary CT angiography: The ERASIR study. AJR Am J Roentgenol 2010;195:655-60.

11. Neroladaki A, Botsikas D, Boudabbous S, Becker CD, Montet X Computed tomography of the chest with model-based iterative reconstruction using a radiation exposure similar to chest X-ray examination: Preliminary observations. Eur Radiol 2013; 23:360-6.

12. Xu Y, He W, Chen H, Hu Z, Li J, Zhang T. Impact of the adaptive statistical iterative reconstruction technique on image quality in ultra-low-dose CT. Clin Radiol 2013;68:902-8.

13. Willemink MJ, Borstlap J, Takx RA, et al. The effects of computed tomography with iterative reconstruction on solid pulmonary nodule volume quantification. PLoS One 2013;8:e58053.

14. Yanagawa M, Honda O, Kikuyama A, et al. Pulmonary nodules: Effect of adaptive statistical iterative reconstruction (ASIR) technique on performance of a computer-aided detection (CAD) system-comparison of performance between different dose CT scans. Eur J Radiol 2012;81:2877-86.

15. Singh S, Kalra MK, Gilman MD, et al. Adaptive statistical iterative reconstruction technique for radiation dose reduction in chest CT: A pilot study. Radiology 2011;259:565-73.

16. Prakash P, Kalra MK, Ackman JB, et al. Diffuse lung disease: CT of the chest with adaptive statistical iterative reconstruction technique. Radiology 2010;256:261-9.

17. Nishio M, Matsumoto S, Ohno Y, et al. Emphysema quantification by low-dose CT: Potential impact of adaptive iterative dose reduction using $3 \mathrm{D}$ processing. AJR Am J Roentgenol 2012;199:595-601.

18. Mets OM, de Jong PA, van Ginneken B, Gietema HA, Lammers JW. Quantitative computed tomography in COPD: Possibilities and limitations. Lung 2012;190:133-45. 


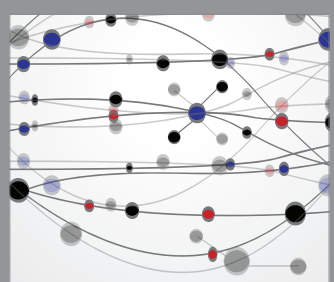

The Scientific World Journal
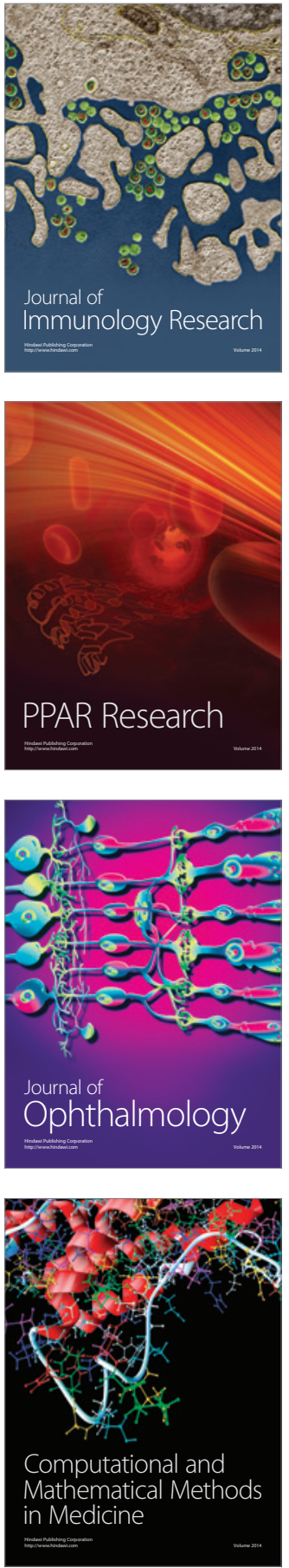

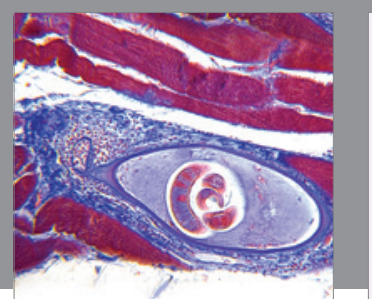

Gastroenterology Research and Practice

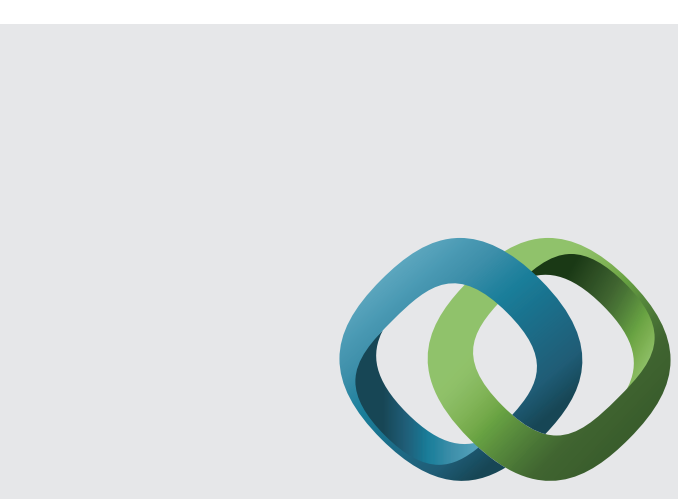

\section{Hindawi}

Submit your manuscripts at

http://www.hindawi.com
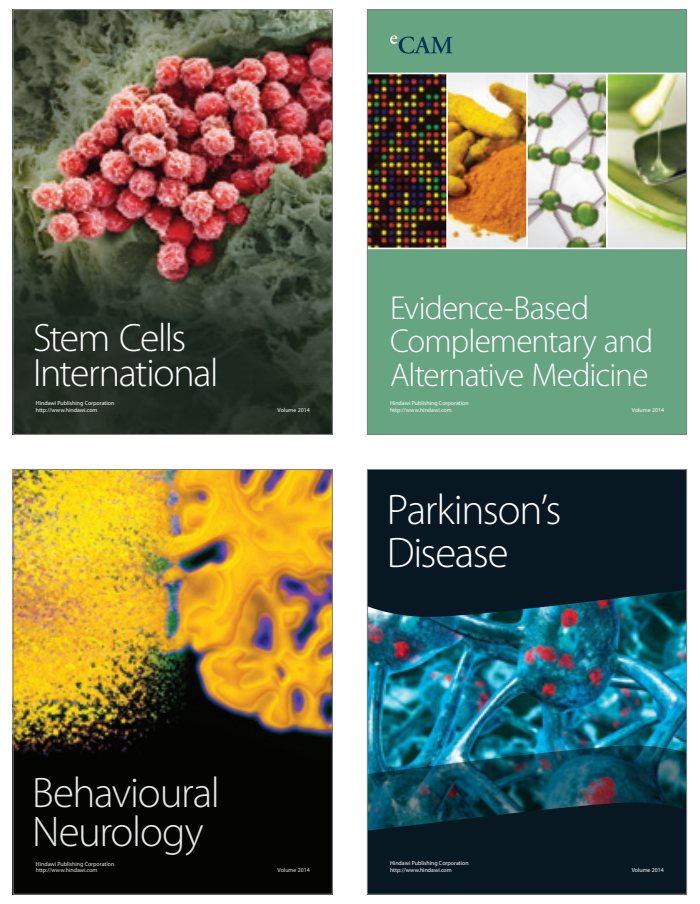
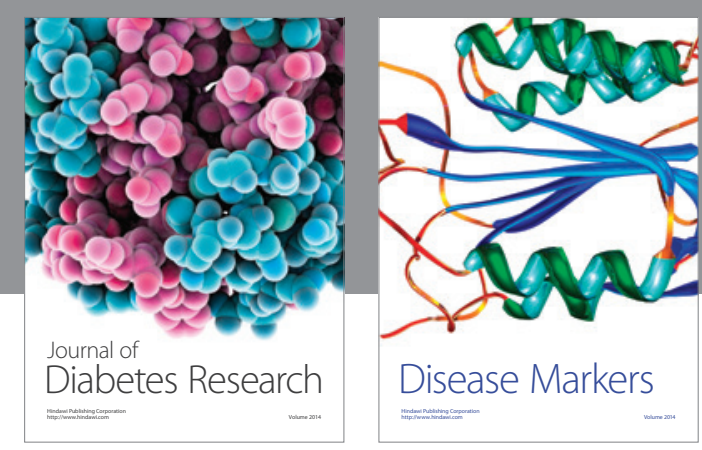

Disease Markers
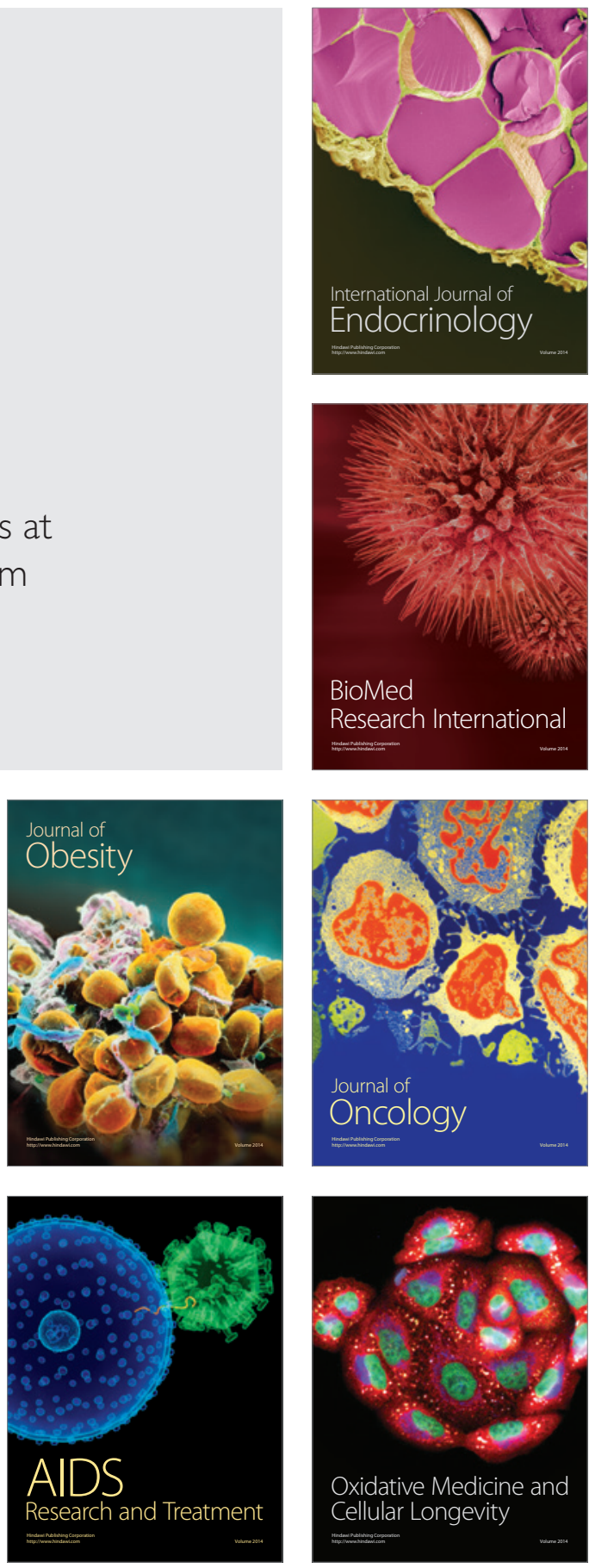